\title{
Design and Simulation of MEMS Piezoresistive Pressure Sensor to Improve the Sensitivity
}

\author{
U. Sampath Kumar ${ }^{1}$, N. Jagadesh Babu² \\ Post Graduate, Dept. of Electronics and Instrumentation Engineering, Gitam University, Visakhapatnam, India ${ }^{1}$ \\ Assistant Professor, Dept. of Electronics and Instrumentation Engineering, Gitam University, Visakhapatnam, India ${ }^{2}$
}

\begin{abstract}
The Piezoresistive pressure sensor presented in this paper describes the optimum techniques to improve the performance of the sensor. Finite element analysis is applied as a part to the design to obtain the results approximately to that of theoretical values. The simulation is carried out considering the membrane geometry size, shape and location of piezoresistors. The applied pressure is transformed into voltage by the piezoresistors which are connected in the form of Wheatstone bridge. The sensitivity of the sensor can be enhanced by selecting proper membrane geometry and the piezoresistors location and in the same way the results are obtained.
\end{abstract}

Keywords: Piezoresistors, MEMS, Piezoresistivity, Pressure sensor.

\section{INTRODUCTION}

\subsection{MEMS Piezoresistive sensors:}

MEMS pressure sensors have changed the way that system designers and application engineers measure pressure. The simplicity of use, small size, low cost and ruggedness allow these sensor to address application in automobile and industrial process control as well as medical and hand held portable products.

Piezoresistivity is common sensing principal for micro machined sensors. Micro pressure sensors are one of the widely used Piezoresistive pressure sensor. Most of this sensor function on the principle of mechanical deformation and stress of thin diaphragms induced by the measurand pressure. The piezoresistors convert the stress induced in the diaphragm by the applied pressure into a change of electrical resistance which is then converted into voltage output by a Wheatstone bridge circuit.

Piezoresistive sensors are made from semiconductors materials usually silicon, with boron as the trace impurity for the P-type material and arsenic as the trace impurity for n-type material. The resistance of a semiconductor Silicon changes as a function of strain. Silicon is therefore a true piezoresistor. The resistivity of a semiconductor material depends on the mobility of charge carrier. The formula for mobility is

$$
\mu=\frac{q \bar{t}}{m^{*}}
$$

Where $\mathrm{q}$ - charge per unit charge carrier

$\bar{t}$ - mean free time between carrier collision events

$m^{*}$-Effective mass of carrier in the crystal lattice

The resistance value of a resistor with the length (l) and the cross-section area (A) is given by

$$
\mathrm{R}=\rho \frac{l}{A}
$$

If length of piezoresistor increases cross-section likely decreases under finite poison's ratios. The change in the resistance as induced by the applied measurand pressure are measured from the Wheatstone bridge in the dynamic deflection operation mode as

$$
V_{0}=V_{\text {in }}\left(\frac{R_{1}}{R_{1}+R_{4}}-\frac{R_{3}}{R_{2}+R_{3}}\right)
$$

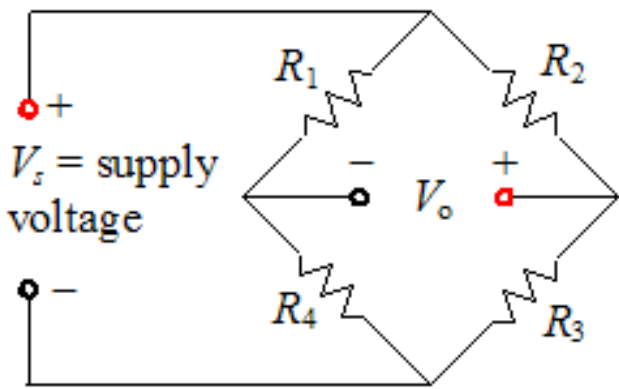

Where $R_{1}, R_{2}, R_{3}$ and $R_{4}$ are resistors coefficients $V_{s}$ - Input voltage $V_{o}$ - Output voltage

\subsection{Finite Element Analysis:}

Finite element analysis (FEA) is playing a critical role in solving many complex problems. Finite element code are less complicated than many of the word processing and spreadsheet packages found on modern microcomputers. In early years Analytical methods are used in solving complex geometrical problems. Later Numerical methods are adopted in solving this complex problems easily.

This numerical methods are further divided into three types:

1. Finite element method

2. Finite difference method

3. Boundary element method

Out of this three methods Finite element method is widely adopted for predicting stress, thermal effect reduction, packaging design and increase the reliability of piezoresistors.

In practice, a finite element analysis usually consists of three principal steps. [2]

1.2.1. Preprocessing: the user constructs a model of the part to be analyzed in which the geometry is divided into a number of discrete sub regions, or "elements," connected at discrete points called "nodes." Certain of these nodes will have fixed displacements, and others will have prescribed loads. These models can be extremely time consuming to prepare, and commercial codes via with one 
another to have the most user-friendly graphical Maximum radial stress at edge

"preprocessor" to assist in this rather tedious chore. Some of these preprocessors can overlay a mesh on a preexisting CAD file, here we used COMSOL multiphysics, so that finite element analysis can be done conveniently as part of the computerized drafting and design process.

1.2.2. Analysis: The dataset prepared by the preprocessor is used as input to the finite element code itself, which constructs solves a system of linear or nonlinear algebraic equation.

$$
K_{i j} u_{j}=f_{i}
$$

Where $\mathrm{u}$ and $\mathrm{f}$ are the displacements and externally applied forces at the nodal points. The formation of the $\mathrm{K}$ matrix is dependent on the type of problem being attacked, and this module will outline the approach for truss and linear elastic stress analyses. Commercial codes may have very large elements libraries, with elements appropriate to wide range of problem types.one of FEA's principal advantages is that many problem types can be addressed with the same code, merely by specifying the appropriate element types from the library.

1.2.3. Postprocessing: in the earlier days of finite element analysis, the user would pore through reams of numbers generated by the code, listing displacements and stress at discrete positions within the model.it is easy to miss important trends and hot spots this way, and modern codes use graphical displays to assist in visualizing the results. A typical postprocessor display overlays colored contours representing stress levels on the model. Showing a fullfilled picture similar to that of photo elastic or moire experimental results.

\section{CALCULATION}

\subsection{Formulas 1}

\subsubsection{Square Diaphragm}

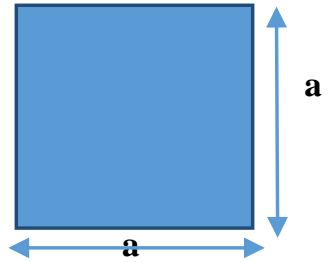

Maximum stress at the center of each Edge

$$
\sigma_{\max }=\frac{0.308 p a^{2}}{h^{2}}
$$

Maximum deflection at the center of the plate

$$
w_{\text {max }}=-\frac{0.0138 p a^{2}}{E h^{3}}
$$

Stress at the center of the plate

\[ \sigma=\frac{\left(6 p(m+1) a^{2}\right.}{47 m h^{2}} \]
Strain at the center $\varepsilon=\frac{1-v}{E} \sigma$

\subsubsection{Circular Diaphragm}

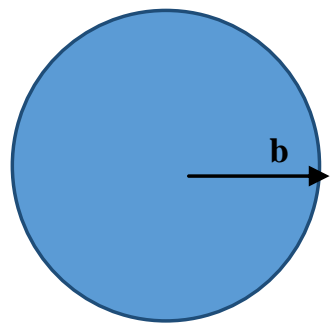

$$
\left(\sigma_{r r}\right)_{\max }=\frac{3 \mathrm{~W}}{4 \pi h^{2}}
$$

Maximum tangential stress at edge

$\left(\sigma_{\Theta \Theta}\right)_{\max }=\frac{3 v W}{4 \pi h^{2}}$

Maximum stress at the center of the diaphragm

$$
\left(\sigma_{r r}\right)_{\max }=\left(\sigma_{\Theta \Theta}\right)_{\max }=\frac{3 v W}{8 \pi h^{2}}
$$

Maximum Deflection at the center of the plate

$$
w_{\text {max }}=-\frac{3 W\left(m^{2}-1\right) b^{2}}{16 \pi E m^{2} h^{3}}
$$

Where $\mathrm{a}=$ Side length of square plate

$\mathrm{b}=$ radius of Circular plate

$\mathrm{h}=$ Thickness of the plate

$\frac{1}{m}=v=$ Poisson's ratio

$\mathrm{E}=$ Young's Modulus

$\mathrm{W}=$ Equivalent load

Note: The dimensions are taken in the form that both the plates area is almost equal, so we can compare results for unit area.

\begin{tabular}{|c|c|}
\hline Type & Dimensions(mm) \\
\hline Square & 2(radius) \\
\hline Circular & 3.5(Sides) \\
\hline
\end{tabular}

Table 1: Dimensions considered for Square and Circular Diaphragms.

\section{INTRODUCED MODELS}

All this models are simulated using Comsol Multiphysics and the results are compared with theoretical results which are nearly equal.

It can observed from the figure that the deflection of circular diaphragm is more compared to square diaphragm.

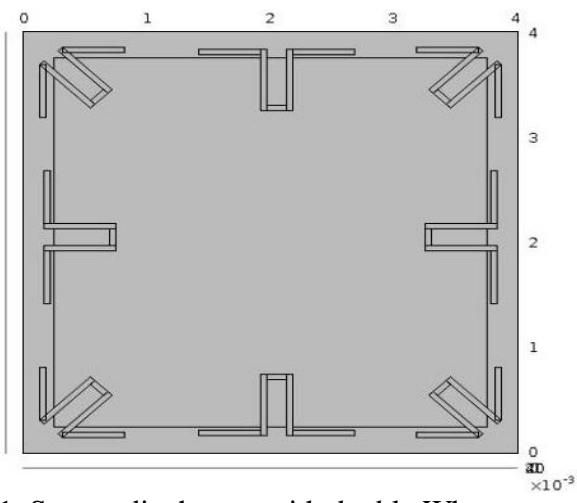

Figure 3.1: Square diaphragm with double Wheatstone bridge

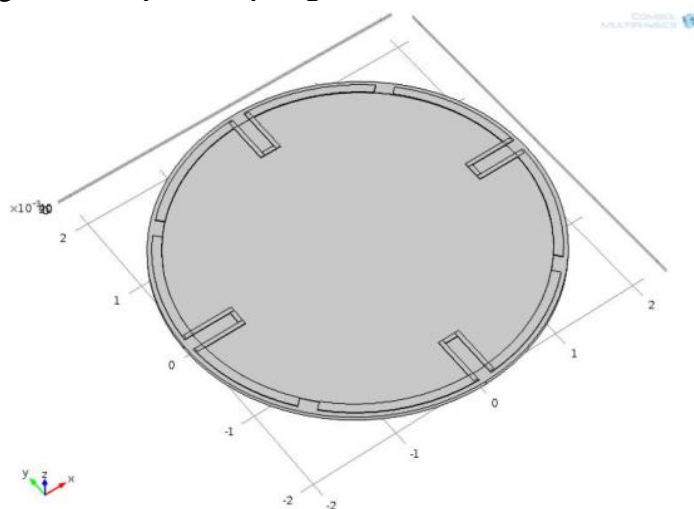

Figure 3.2: Circular Diaphragm with Single Wheatstone Bridge 


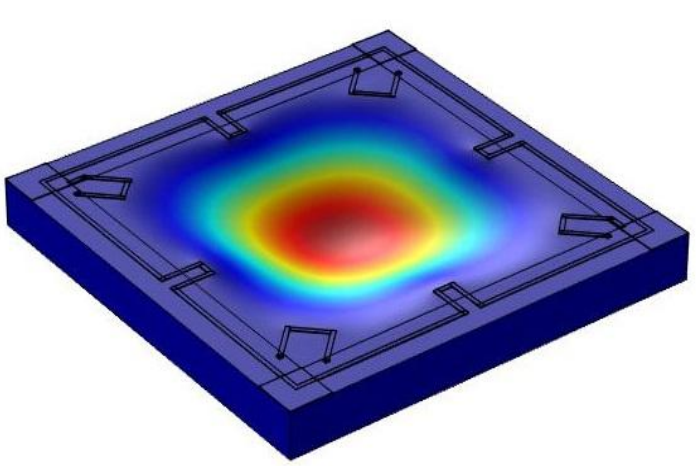

Figure 3.4: Deflection of square diaphragm

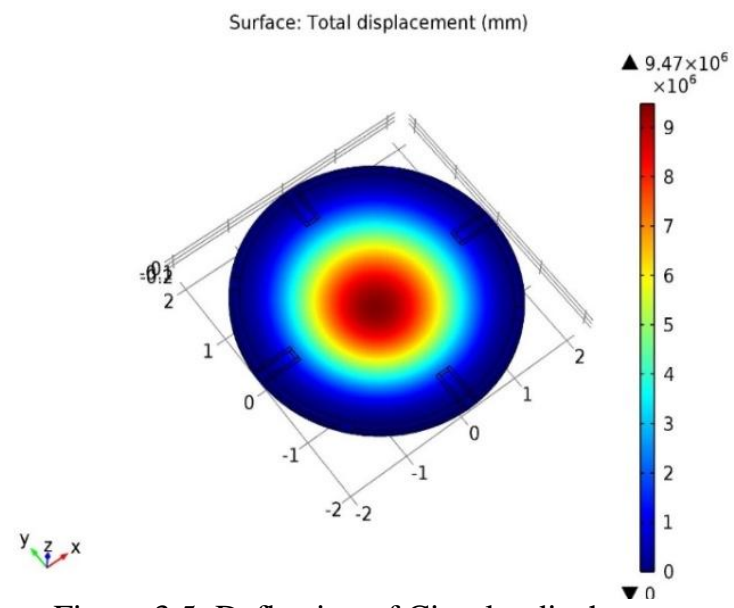

Figure 3.5: Deflection of Circular diaphragm

\section{ANALYSIS ON SIMULATIONS}

It is observed from the results that the deflection of the membrane is minimum at sides and maximum at the edges. So to use this maximum deflection area effectively another Wheatstone bridge is implanted at the edges. This makes two Wheatstone bridges in the sensor. By using double Wheatstone bridge the voltage sensitivity of the Piezoresistive pressure sensor is increases.

In this model the number of resistors are doubled to increase the sensitivity of sensor. So to reduce the number of resistors and simultaneously increase the sensitivity of the sensor the geometry is changed to Circle in which the stress is distributed equally on all directions.

\section{RESULTS}

\begin{tabular}{|c|c|c|}
\hline Type & Stress $\left(\mathbf{N} / \boldsymbol{M}^{\mathbf{2}}\right)$ & Deflection(mm) \\
\hline Square & 756.6 & 0.2179 \\
\hline Circular & 602.88 & 0.29605 \\
\hline
\end{tabular}

Table 5.1: Maximum Deflections vs. Stress between Square and Circular diaphragm

From the above table it is analyzed that the circular diaphragm exhibits maximum deflection compared to the square diaphragm and the stress taken by circular diaphragm is much less than the square diaphragm. Due to single Wheatstone bridge the sensor output voltage is less compared to double Wheatstone bridge square diaphragm. A graph is plotted using above table values and it is observed that circular diaphragm is deflecting more compared to square diaphragm.

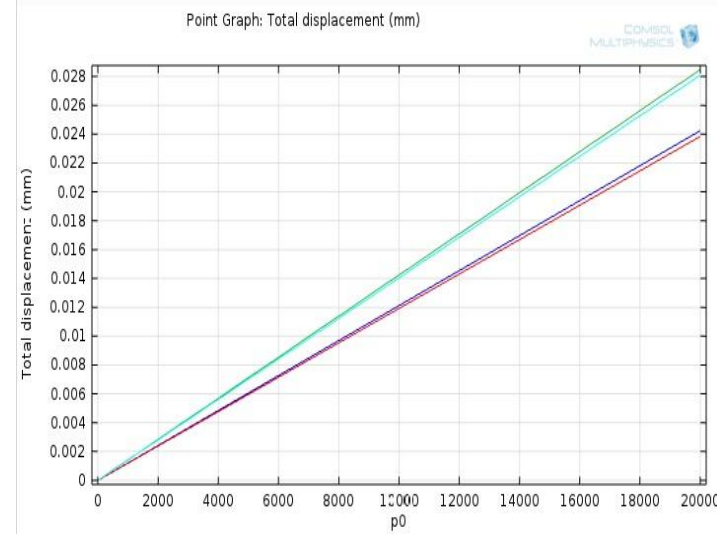

Figure 5.1: Comparison of deflection Vs. Pressure for square and circular diaphragm

From the simulations and theoretical results it is observed that the effective use of membrane deflection area by using double Wheatstone bridge can increase the sensitivity of the sensor.

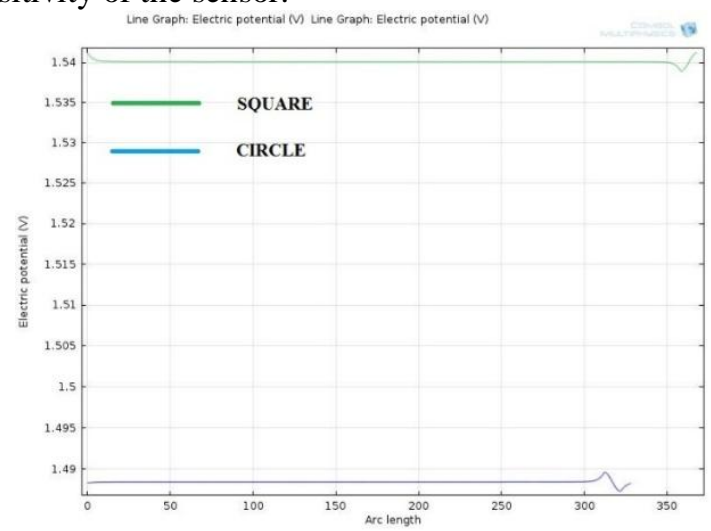

Figure 5.2 Electric Potential caused in Square and circular Diaphragm From the figure it is observed that the Electrical potential is more in Square diaphragm compared to circular diaphragm. So the sensitivity can be increased by placing double Wheatstone bridge rather than changing the geometry.

And coming to the fabrication process square membrane are existing techniques and costs less when compared to circular membrane as the design of layouts cost more for circular layouts which indeed increases the cost of the sensor.

\section{CONCLUSION}

From the above analysis and results it is observed that effective use of sensor deflection areas can increase the sensitivity of the sensor. In this model the sensitivity can be increased by placing another Wheatstone bridge at the corners.

\section{REFERENCES}

"MEMS \& Microsystems Design and manufacture" by Tai-Ran Hsu.

"Finite Element Analysis" by David Roylance, MIT.

"Differential Piezoresistive Pressure sensor" by B. Firtat, C.Moldovan , R.Iosub , National Institute of Research and Development in Microtechnologies, IEEE 2007

4. "Performance Analysis of Piezoresistive MEMS for Pressure measurement". MZ.Shaikh, Dr.S.F.Kodad, Research scholar, JNTU Hyderabad.

5. "Analysis and Validation of Sensing Sensitivity of a Piezoresistive pressure Sensor" by Chih-Tang Peng, Ji-Cheng Lin, National Tsing Hua University.

6. Design and characterization of a MEMS Piezoresistive Cochlearlike acoustic sensor by R White and K. Grosh, IMECE 2002. 